\title{
Standardized Protocol for Endodontic Treatment (Iraqi Endodontic Society)
}

\author{
Raghad A. Al-Hashimi \\ BDS, MSc, PhD (UK), Assistant professor \\ Hussain F. Al-Huwaizi \\ BDS, MSc, PhD, Professor
}

\begin{abstract} has been designed in broad terms.

\section{CITE THIS ARTCLE:} 37(2):69-72. http://www.iraqidentaljournal.com

\section{INTRODUCTION}

Endodontology is concerned with the study of the form, function and health of, injuries to and diseases of the dental pulp and periradicular region, their prevention and treatment; the principle disease being apical periodontitis, caused by infection (European Society of Endodontology 2006). Endodontic treatment involves procedures that are designed to preserve the health of all or part of the dental pulp. When the dental pulp is diseased or injured, treatment is targeted to maintaining normal periradicular tissues by creating a favorable biological environment to facilitate the healing process of the diseased periradicular tissue (European society of endodontology 2001).
\end{abstract}

The establishment of a high quality service in oral health care achieved by a member of dental professional is an important feature of any system of peer review in dentistry. This protocol attempts to discuss two crucial elements (I) suitability and feasibility of treatment modality and (II) quality of treatment performed to Iraqi patients. The Iraq endodontic society is designing a standardized protocol for endodontic treatment following the quality guidelines of European society of Endodontology (2006) to meet the highest standard of care generally given by competent practitioners. The Iraqi endodontic society has the expertise and professional responsibility clinically relevant to empower the dental profession through creating significant guidelines on the standard of care in the endodontic discipline. Because there is multiple way of performing endodontic treatment, this standardized protocol

Al-Hashimi R, Al-Huwaizi H. Standardized Protocol for Endodontic Treatment (Iraqi Endodontic Society). Iraqi Dent. J. 2015;

\section{HISTORY, DIAGNOSIS AND TREATMENT PLANNING}

Many features of evaluation in Endodontics are common to all aspects of dental practice.

\section{Medical and dental history}

Medical history should reveal any medical condition or medication which might influence diagnosis, e.g. sinusitis, neoplasia, or treatment which may be influenced by dental procedures; this should include allergy. Dental history discovers factors that may be important for diagnosis and treatment planning. History of present complaint is recorded briefly and preferably in the patient's own words. Pain history is recorded to give information on the pain, but phrased to avoid leading questions. The questions may include: the nature, duration, site, periodicity, precipitating or relieving factors and associated symptoms.

\section{Clinical examination}

The patient should be examined both extraand intraorally and may also need to be checked for pyrexia and blood pressure.

\section{I.Extra-oral examination}

The practitioner should look for asymmetry, presence and extent of swelling in the head and neck region, lymphadenopathy, presence of sinus tracts and presence of temporomandibular joint dysfunction.

The practitioner should look for the standard of oral hygiene, condition of oral mucosa, presence of swellings and sinus tracts, condition of teeth present, periodontal condition, quantity and quality of restorative work.

\section{Diagnosis}

The cause of the patient's complaint should be identified. Some or all, of the following diagnostic tests may be applied: palpation, mobility test, percussion, periodontal examination, occlusal analysis, testing for possible cracked teeth, pulp sensitivity tests, transillumination, selective local anaesthesia, radiography (normally by using the paralleling technique and a beam guiding device for good reproducibility), colour matching and sinus tract

\section{II.Intra-oral examination}


exploration. It may be necessary to take radiographs from more than one angle, sometimes supplemented with bitewing and occlusal plane radiographs. Some patients may have to be recalled at periodic intervals to compare some of the examination data from one time interval to another to make an accurate diagnosis of the onset, progression or arrest of a certain process. At times it is advisable to obtain radiographs from previous practitioners to have a clearer understanding of the progress of a condition (as it presents to the clinician at a given time). This is the case especially where previous root canal treatment has been carried out.

\section{Treatment planning}

Treatment should be planned for those teeth that are functionally or aesthetically important and have reasonable prognosis. Procedures to maintain pulp health are described in 'Management of the vital pulp.

\section{INDICATIONS FOR ROOT CANAL TREAT- MENT}

Root canal treatment may be carried out on all patients where other dental procedures may be undertaken.Specific indications are

1. An irreversibly damaged or necrotic pulp with or without clinical and/or radiological findings of apical periodontitis.

2. Elective devitalization, e.g. to provide post space, prior to construction of an overdenture, doubtful pulp health prior to restorative procedures, likelihood of pulpal exposure when restoring a (misaligned) tooth and prior to root resection or hemisection.

\section{CONTRA-INDICATIONS FOR ROOT CA- NAL TREATMENT}

1. Teeth that cannot be made functional nor restored.

2. Teeth with insufficient periodontal support.

3. Teeth with poor prognosis, uncooperative patients or patients where dental treatment procedures cannot be undertaken.

4. Teeth of patients with poor oral condition that cannot be improved within a reasonable period.

\section{INDICATIONS FOR ROOT CANAL RE- TRE A T ME N T}

1. Teeth with inadequate root canal filling with radiological findings of developing or persisting apical periodontitis and/or symptoms.
2. Teeth with inadequate root canal filling when the coronal restoration requires replacement or the coronal dental tissue is to be bleached.

\section{ROOT CANAL TREATMENT}

Root canal treatment is carried out when the pulp is nonvital or has been removed to prevent or treat apical periodontitis. The purpose of root canal treatment is either to maintain asepsis of the root canal system or to disinfect it adequately.

\section{a.Preoperative radiograph}

A preoperative radiograph showing at least the full root(s) and approximately $2-3 \mathrm{~mm}$ of periapical region, must be examined prior to treatment.

\section{b.Local anaesthesia}

The need for local anaesthesia should be considered and given as appropriate.

\section{c.Preparation of tooth}

All caries and defective restorations should be removed and, if necessary, the occlusion adjusted and the tooth protected against fracture. The tooth should be capable of being restored and isolated and the periodontal status should be sound or capable of resolution.

\section{d.Isolation of tooth}

Root canal treatment procedures should be carried out only when the tooth is isolated by rubber dam to:

Prevent salivary and bacterial contamination, prevent inhalation and ingestion of instruments and prevent irrigating solutions escaping into the oral cavity.

\section{e.Access cavity preparation}

The objectives of the access cavity preparation are to: remove the roof of the pulp chamber so that this

chamber can be cleaned and good visibility of the canal orifices can be obtained, enable root canal instruments to be introduced into the root canal(s) without undue bending, offer sufficient retention for a temporary restoration and conserve as much sound tooth tissue as possible that is compatible with the above.

f.Determination of working length 
The objective of determining the working length is to enable the root canal to be prepared as close to the apical constriction as possible. The location of the apical constriction normally varies between 0.5 and $2 \mathrm{~mm}$ from the radiographic apex. Recommended methods are electronic and radiographic.

\section{Electronic}

Electronic devices measure the length of the root canal accurately in most cases. The working length should normally be confirmed radiographically.

\section{Radiographic}

The instrument should be equipped with some form of length indicator and be of sufficient size so that its tip can be clearly identified on the radiograph. A radiograph is then taken which should show the instrument and the apex with minimal distortion. The desired working length is determined. If the distance between the tip of the instrument and the desired working length is $>3 \mathrm{~mm}$, the working length of the file is adjusted and a further radiograph taken. It may be necessary to take more than one radiograph.

\section{g.Preparation of the root canal system}

The objectives of preparation are to: remove remaining pulp tissue, eliminate microorganisms, remove debris and shape the root canal(s) so that the root canal system can be cleaned and filled. The use of magnification and additional sources of light facilitate identification of root canal anatomy. The requirements should be: the prepared canal should include the original canal, the apical constriction should be maintained, the canal should end in an apical narrowing and the canal should be tapered from crown to apex. Preparation should be undertaken with copious irrigation.

\section{h.Irrigation}

The objectives of irrigation are to: eliminate microorganisms, flush out debris, lubricate root canal instruments and dissolve organic debris. The irrigant solution should preferably have disinfectant and organic debris dissolving properties, whilst not irritating the periradicular tissues. The irrigant solution should be delivered in copious amounts as far up the canal as possible without risking extrusion beyond the foramen. This can be performed with a syringe, ensuring that the solution is allowed to escape freely into the pulp chamber and is not delivered with excessive force. The solution may also be delivered by ultrasonic or sonic systems.
i.Inter-appointment medication

The objectives of inter-appointment medication are to prevent growth and multiplication between visits of microorganisms left in the canal system despite cleaning. They should be used following proper cleaning and irrigation and to support the tissue-dissolving effects of irrigating solutions. This stage is rarely necessary after pulpectomy and root canal preparation of a tooth with a vital pulp. An effective temporary restoration is essential to prevent contamination of the canal system between visits. The requirements of an interappointment disinfectant are: have long-lasting disinfectant action, be biocompatible, be removable and be non-damaging to tooth structure or the restorative material.

\section{j.Filling of the root canal system}

The objectives are: to prevent the passage of microorganisms and fluid along the root canal and to fill the whole canal system, not only to block the apical foramina but also the dentinal tubules and accessory canals. Materials used to fill the root canal system should be: biocompatible, dimensionally stable, able to seal, unaffected by tissue fluids and insoluble, nonsupportive of bacterial growth, radiopaque, and removable from the canal if retreatment needed. The root canal filling should consist of a (semi-) solid material in combination with a root canal sealer to fill the voids between the (semi-) solid material and root canal wall. Sealers containing organic materials such as aldehydes are not recommended. Filling should be undertaken after the completion of root canal preparation and when the infection is considered to have been eliminated and the canal can be dried. In some cases it might be recommended that prior to obturation, the completion of root canal preparation is verified by taking a radiograph with the root canal instrument(s) (or filling cones) inserted to the full working length. The end-point of the inserted instrument (or cone) and the apex should be visible on this verification radiograph.

The quality of filling should be checked with a radiograph. This radiograph should show the root apex with preferably at least $2-3 \mathrm{~mm}$ of the periapical region clearly identifiable. The prepared root canal should be filled completely unless space is needed for a post. The prepared and filled canal should contain the original canal. No space between canal filling and canal wall should be seen. There should be no canal space visible beyond the end-point of the root canal filling. The tooth should be adequately restored after 
root canal filling to prevent bacterial recontamination of the root canal system or fracture of the tooth.

\section{PRIMARY ROOT CANAL TREATMENT PR OCEDURE}

Briefly, the entire procedure is undertaken after isolation of the tooth using a rubber dam. The existing restorations and any caries are removed completely. Then, the root canal orifice is opened and A hand instrument K-flexofile size 10 (Dentsply Maillefer) is used until full working length is achieved and confirmed by apex locater (Root ZX, J. Morita, Osaka, Japan) and periapical radiograph. Instrumentation of the root canal is completed with NiTi hand or rotary based endodontic system ProTaper universal files (Dentsply Maillefer) S1 to F2 or F3 or waveone (Densply Maillfer) small or primary or large to the full working length with passive ultrasonic irrigation with $2 \%$ sodium hypochlorite. Calcium hydroxide $\mathrm{Ca}(\mathrm{OH})_{2}$ dressing is placed inside root canals in between treatment visits. Finally, the root canal should be filled with permanent root canal filling material matching the instrumentation system used.

\section{SECONDARY ROOT CANAL TREATMENT (RETREATMENT) PROCEDURE}

Briefly, the entire procedure is undertaken after isolation of the tooth using a rubber dam and using an operating microscope for all the clinical procedures. The existing restorations, and any caries are removed completely and Gates-Glidden drills (Dentsply Maillefer) sizes 2 or 3 at $600 \mathrm{rpm}$ are used to clear the coronal gutta-percha. Then, the root canal orifice flooded with little amount of chloroform. ProTaper Retreatment files ${ }^{\circledR}$ (Dentsply Maillefer, Ballaigues, Switzerland) D2 and D3 are used to remove the old gutta-percha from the middle and apical third of thecanal respectively. A hand instrument K-flexofile size 10 (Dentsply Maillefer) is used until full working length is achieved and confirmed by apex locater (Root ZX, J. Morita, Osaka, Japan) and periapical radiograph. Re-instrumentation is completed with ProTaper universal files (Dentsply Maillefer) S1 to F2 or F3 to the full working length with passive ultrasonic irrigation with $2 \%$ sodium hypochlorite and a penultimate irrigation with $17 \%$ EDTA. Calcium hydroxide $\mathrm{Ca}(\mathrm{OH}) 2$ dressing is placed inside root canals in between treatment visits. All root canal re-treatments are performed in two visits.

\section{NAL TREATMENT}

The primary purpose of outcome assessment is to monitor healing or development of pulpitis or apical periodontitis. Clinical and radiographic follow-ups at regular intervals for a minimum observation period of 1 year are desirable, but longer may be required where healing is incomplete or there is a history of trauma. A favourable outcome may be reversed if infection or reinfection occurs.

Root canal treatment should be assessed at least after 1 year and subsequently as required. The following findings indicate a favourable outcome: absence of pain, swelling and other symptoms, no sinus tract, no loss of function and radiological evidence of a normal periodontal ligament space around the root. Root canal treatment has an uncertain outcome If radiographs reveal that a lesion has remained the same size or has only diminished in size, the outcome is considered uncertain. In this situation it is advised to assess the lesion further until it has resolved or for a minimum period of 4 years. If a lesion persists after 4 years the root canal treatment is usually considered to be associated with post-treatment disease. Root canal treatment has an unfavourable outcome. This occurs when

1. The tooth is associated with signs and symptoms of infection.

2. A radiologically visible lesion has appeared subsequent to treatment or a pre-existing lesion has increased in size.

3. A lesion has remained the same size or has only diminished in size during the 4-year assessment period.

4. Signs of continuing root resorption are present. In these situations it is advised that the tooth requires further treatment.

Exception: An extensive radiological lesion may heal but leave a locally visible, irregularly mineralized area. This defect may be scar tissue formation rather than a sign of persisting apical periodontitis. The tooth should continue to be assessed.

\section{REFERENCES}

1. European Society of Endodontology (2006) quality-quidelines for endodontic treatment. International Endodontic Journal 39, 921-930.

2. European Society of Endodontology (2001) Undergraduatecurriculum guidelines for endodontology. International Endodontic Journal 34, 574-80. 